\title{
LiveWall Operational Evaluation: Seattle Law Enforcement Pilot
}

\author{
JL Barr \\ ER Burtner \\ SL Stein
}

October 2013

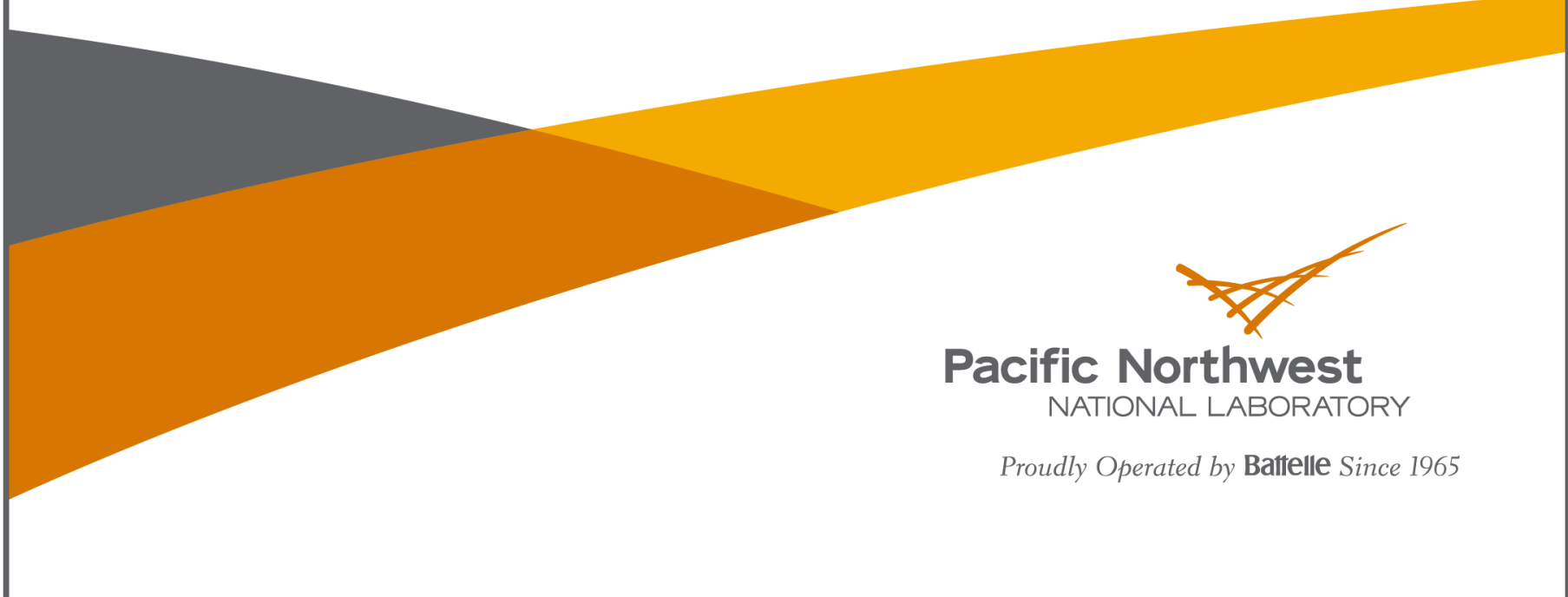




\title{
DISCLAIMER
}

This report was prepared as an account of work sponsored by an agency of the United States Government. Neither the United States Government nor any agency thereof, nor Battelle Memorial Institute, nor any of their employees, makes any warranty, express or implied, or assumes any legal liability or responsibility for the accuracy, completeness, or usefulness of any information, apparatus, product, or process disclosed, or represents that its use would not infringe privately owned rights. Reference herein to any specific commercial product, process, or service by trade name, trademark, manufacturer, or otherwise does not necessarily constitute or imply its endorsement, recommendation, or favoring by the United States Government or any agency thereof, or Battelle Memorial Institute. The views and opinions of authors expressed herein do not necessarily state or reflect those of the United States Government or any agency thereof.

\author{
PACIFIC NORTHWEST NATIONAL LABORATORY \\ operated by \\ BATTELLE \\ for the \\ UNITED STATES DEPARTMENT OF ENERGY \\ under Contract DE-AC05-76RL01830 \\ Printed in the United States of America \\ Available to DOE and DOE contractors from the \\ Office of Scientific and Technical Information, \\ P.O. Box 62, Oak Ridge, TN 37831-0062; \\ ph: (865) 576-8401 \\ fax: $(865) 576-5728$ \\ email: reports@adonis.osti.gov \\ Available to the public from the National Technical Information Service \\ 5301 Shawnee Rd., Alexandria, VA 22312 \\ ph: (800) 553-NTIS (6847) \\ email: orders@ntis.gov <http://www.ntis.gov/about/form.aspx> \\ Online ordering: http://www.ntis.gov
}

This document was printed on recycled paper. 


\section{LiveWall Operational Evaluation: Seattle Law Enforcement Pilot}

JL Barr

ER Burtner

SL Stein

October 2013

Prepared for

the U.S. Department of Homeland Security under a Government Order with the U.S. Department of Energy

Contract DE-AC05-76RL01830

Pacific Northwest National Laboratory

Richland, Washington 99352 


\section{Summary}

First responders rely heavily on technology to increase their situational awareness while at an incident, monitoring an incident from afar, or conducting day-to-day response. It is critical for emergency operations centers, field operations sites, state and local law enforcement, and fire and rescue to communicate quickly and efficiently when responding to routine or emergency events. The LiveWall project improves inter- and cross-agency coordination by giving agencies a life-size, always-available, digital "portal" into other locations.

For the initial LiveWall operational evaluation, Pacific Northwest National Laboratory (PNNL) partnered with the Seattle Police Department (SPD). The hardware deployed for the operational evaluation was the InFocus INF5520 MondoPad ${ }^{\mathrm{TM}}$. The MondoPad consists of a number of integrated components, including PC, touchscreen, HD camera, stereo microphone, and speakers.

PNNL and SPD collaborated on three LiveWall testing modes that demonstrated the greatest benefit to SPD for their work during the Torchlight Parade, city-wide celebration held in downtown Seattle which is associated with the SeaFair summer festival. The parade attracts more than 300,000 people. The LiveWall system was used to support communicating the commander's intent, expedite roll call while improving accuracy, and improve situational awareness across SPD during the Torchlight Parade. PNNL embedded observers with various user groups to observe and evaluate the LiveWall concept as implemented in the SPD operations.

The Seattle Law Enforcement Pilot for the LiveWall concept was deemed a success from both the PNNL evaluators and the stakeholders from SPD (operations, intelligence, and information technology). The system demonstrated the ability to

- substantially reduce amount of time for various tasks as seen when disseminating commander's intent, performing roll call, disseminating arrest information to operations and public information officer

- improve accuracy with which information is transferred as seen in disseminating the commander's intent and jointly working through roll call

- support improved situation awareness as seen when collaborating on potential threats.

SPD is planning on keeping the LiveWall systems installed in operations and is working to use the systems in more day-to-day activities such as briefings and other meetings. SPD staff felt that the more familiarity that users had with the system, the more they would be comfortable when using it and understanding the strengths it affords. 



\section{Acronyms and Abbreviations}

$\begin{array}{ll}\text { A/V } & \text { audio visual } \\ \text { EOCs } & \text { emergency operations centers } \\ \text { HD } & \text { high definition } \\ \text { ITS } & \text { Information Technology Section (of the Seattle Police Department } \\ \text { MondoPad } & \text { InFocus INF5520 MondoPad }{ }^{\mathrm{TM}} \text { (and its integrated components) } \\ \text { PC } & \text { personal computer } \\ \text { PIE } & \text { Precision Information Environment } \\ \text { PNNL } & \text { Pacific Northwest National Laboratory } \\ \text { SPD } & \text { Seattle Police Department }\end{array}$





\section{Contents}

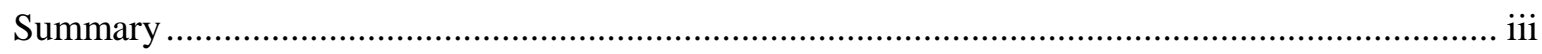

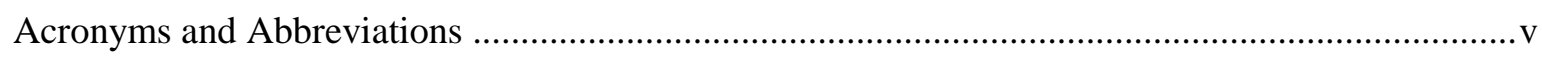

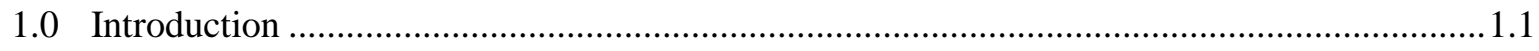

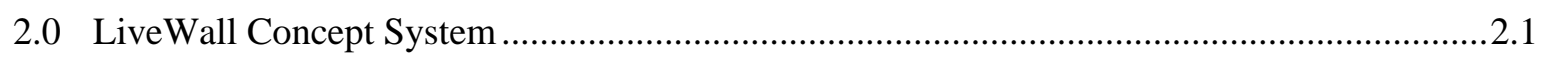

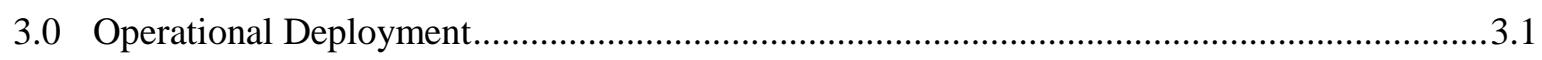

3.1 Integrating the System into Operations ................................................................... 3.1

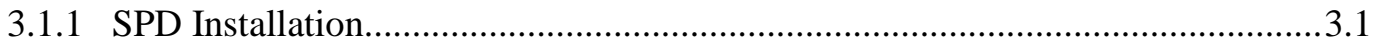

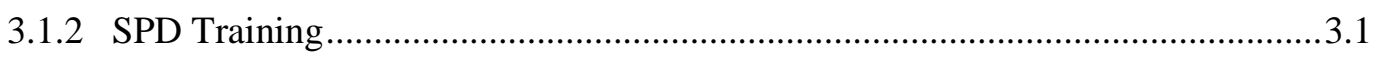

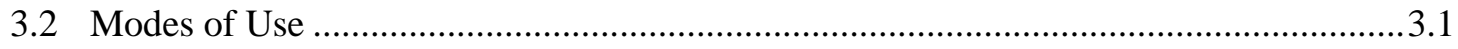

3.2.1 Commander's Intent and Torchlight Briefing .................................................... 3.2

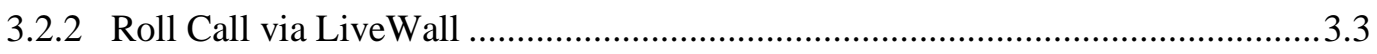

3.2.3 Situational Awareness and Steady State Operations ............................................. 3.5

4.0 Operational Evaluation of Law Enforcement Use Cases..................................................... 4.1

4.1 Commander's Intent and Torchlight Briefing ......................................................... 4.1

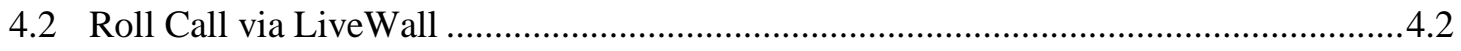

4.3 Situation Awareness and Steady State Operations ...................................................... 4.3

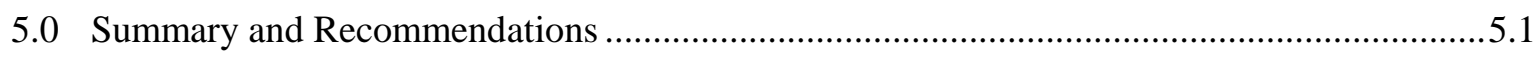




\section{Figures}

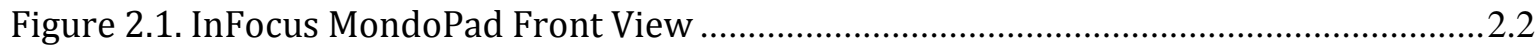

Figure 3.1. LiveWall-based Workflow for Commander's Intent .............................................3.3

Figure 3.2. LiveWall-based Workflow for Roll Call ................................................................. 3.5

Figure 3.3. Seattle Operations Staff Annotating a Shared Map on a MondoPad...........................3.6

Figure 3.4. LiveWall-based Workflow for Situational Awareness ................................................. 3.7

Figure 4.1. Incident Commander Shown Simultaneously Briefing Commander's Intent with LiveWall System to Officers at Local and Remote Locations................................................ 4.2 


\subsection{Introduction}

First responders rely heavily on technology to increase their situational awareness while at an incident, monitoring an incident from afar, or conducting day-to-day response. It is critical for emergency operations centers (EOCs), field operations sites, state and local law enforcement, and fire and rescue to communicate quickly and efficiently when responding to routine or emergency events. The LiveWall project improves inter- and cross-agency coordination by giving agencies a life-size, always-available, digital "portal" into other locations.

The LiveWall concept, envisioned as an outgrowth of the Precision Information Environment (PIE) project, makes possible communications among separate groups using interactive video, audio, and a shared desktop environment - this allows everyone to participate and collaborate in real time, regardless of location. The LiveWall concept provides a virtual window to other locations, where all parties can interact and collaboratively work with each other. This functionality is intended to improve multi-site coordination among EOCs, field operations sites, and across organizations and jurisdictions to accommodate communications during routine and emergency events.

For the initial LiveWall operational evaluation, Pacific Northwest National Laboratory (PNNL) partnered with the Seattle Police Department (SPD). This partnership allowed the creation of an excellent LiveWall test bed specific to law enforcement. PNNL and SPD agreed that integrating the systems into operations for a real event would be the best test of the technology and give SPD staff greater visibility into the functionality and benefits offered by the LiveWall concept.

One of SPD's major deployment events, Torchlight Parade, was selected for the operational setting for the LiveWall evaluation. The Torchlight Parade is a major city-wide celebration associated with the SeaFair summer festival. The parade is held in downtown Seattle and attracts more than 300,000 people. It is critical that SPD ensures public safety at an event of this size, which requires that all officers know what their role and mission are, that resources are available to accomplish that mission, and that the information that needs to be acted on is quickly and effectively communicated to achieve situational awareness.

PNNL and SPD collaborated on three LiveWall testing modes that demonstrated the greatest benefit to SPD for supporting the Torchlight Parade. The three modes involved using the LiveWall concept to 1) support communicating the commander's intent, 2) expedite roll call while improving accuracy, and 3) improve situational awareness across SPD. Each of these scenarios is detailed in the next section on operational deployment. 


\subsection{LiveWall Concept System}

The hardware deployed for the operational evaluation was the InFocus INF5520 MondoPad ${ }^{\mathrm{TM} 1}$. The InFocus MondoPad consists of a number of integrated components: touchscreen display interface with integrated Microsoft Windows ${ }^{\mathrm{TM} 2}$ based personal computer (PC), high definition (HD) camera and microphone array, and sound bar (Figure 2.1). To integrate these components and extend the functionality of the system, there are a number of connector panels available to allow the system to interface with both primary components (display, HD camera and microphone array, sound bar) and secondary systems (external PC, universal serial bus [USB] mouse/keyboard, external display, external audio, and external video sources) ${ }^{3}$. One of the advantages of using a system such as the MondoPad is the ability to interact directly with the device and add a tactile interaction while working with the system. This is accomplished by using the incorporated touchscreen display and touchscreen keyboard.

The InFocus MondoPad System is capable of using InFocus's MondoPad software for collaboration, as well as any other PC-based collaboration tools (e.g. Microsoft Lync ${ }^{\mathrm{TM} 2}$, Vidtel ${ }^{\mathrm{TM} 4}$, Skype ${ }^{\mathrm{TM} 5}$, Google Hangouts $^{\mathrm{TM} 6}$, etc). SPD selected Cisco's WebEx ${ }^{\circledR}$ platform $^{7}$ as the primary collaboration software for the operational deployment of the systems. SPD was familiar with the software because it is the SPD's current web-conferencing software. The functionality afforded by the WebEx software also allowed for the majority of the desired functionality for the deployment: allowing for multiple parties on a variety of platforms to simultaneously collaborate with audio, video, desktop sharing, white boarding, file sharing, and annotation tools.

Three units mounted on mobile stands were deployed to SPD for this effort. The mobile stands allowed the units to be moved quickly between locations, effectively expanding the footprint of where and how the LiveWall concept was tested.

\footnotetext{
${ }^{1}$ Trademark of the InFocus Corporation, Portland, Oregon

2 Trademark of the Microsoft Corporation, Redmond, Washington.

${ }^{3}$ InFocus. 2013. INF5520 Hardware Guide. Accessed October 14, 2003 at http://www.infocus.com/sites/default/files/SupportDocs/Mondopad_Family_Page/INF5520/INF5520_Hardware_Gu ide.pdf

${ }^{4}$ Trademark of the Vidtel, Inc., Sunnyvale, California

${ }_{6}^{5}$ Registered trademark of Premium Installer, Inc., Fort Myers, Florida.

${ }^{6}$ Trademark of Google, Inc., Mountain View, California

${ }^{7}$ Registered Trademark of Cisco Systems, Inc., San Jose, California
} 


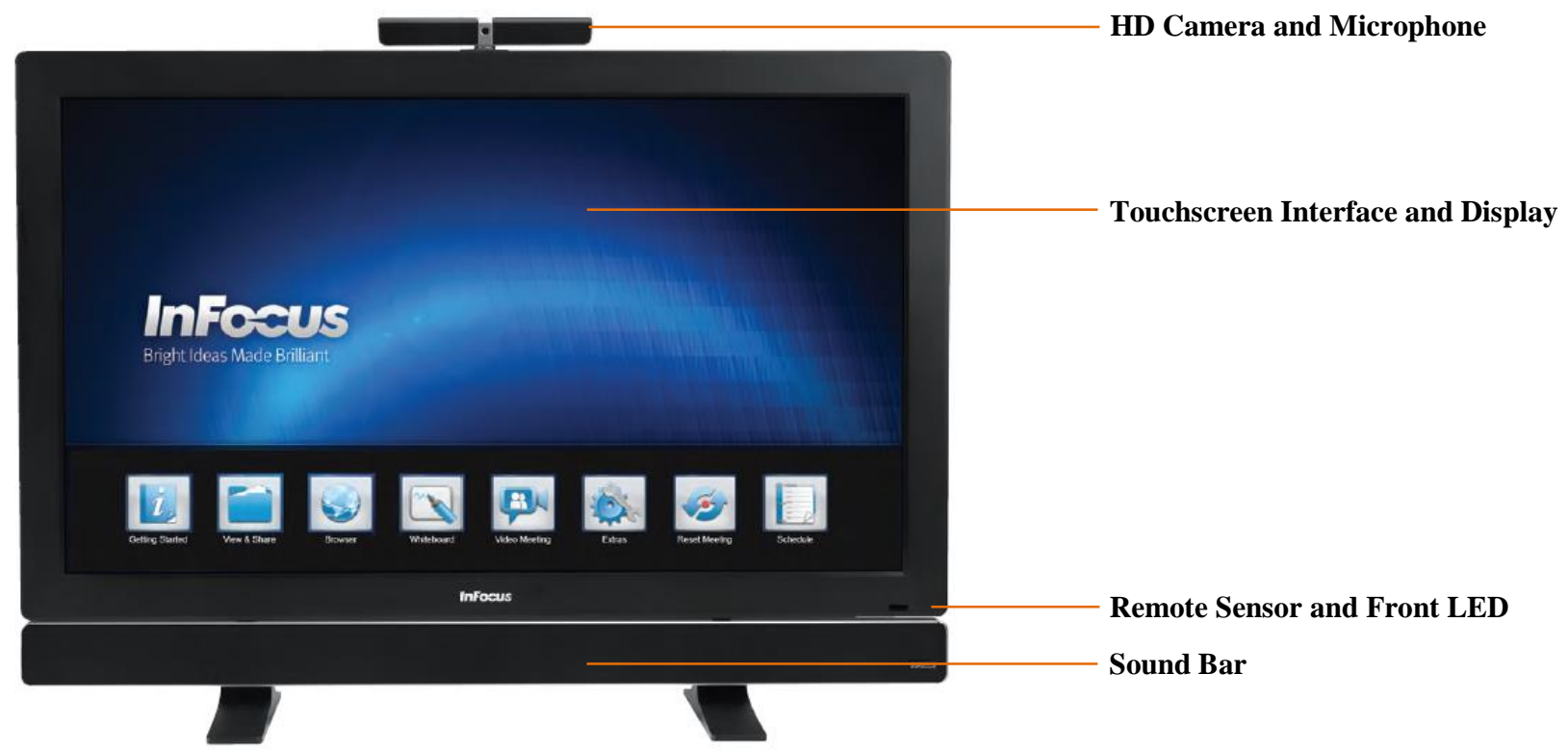

Figure 2.1. InFocus MondoPad Front View 


\subsection{Operational Deployment}

The following sections describe the deployed system, the process used by SPD to integrate the system into operations, and the modes and functionality evaluated during the Torchlight Parade operational deployment.

\subsection{Integrating the System into Operations}

\subsubsection{SPD Installation}

SPD was provided with three assembled InFocus MondoPad Systems each with a wireless keyboard and mouse. SPD Information Technology Section (ITS) staff then configured the Windows operating system of the MondoPads to ensure that the operating system was patched appropriately to match SPD's current system and security configurations. Because the MondoPad is based on a fully functional PC, it was possible to connect to the internal SPD computer network and gather information from other systems and display it on the MondoPad for the operation. For example, SPD IT was able to create and map the required network drives for the Torchlight Parade use cases on each MondoPad (see Section 3.2 for use case descriptions). Finally, SPD-IT installed and tested the Cisco WebEx software and plugins. SPD ITS indicated that "there were no configuration issues with setting up MondoPads with WebEx, just install (WebEx) and go."

\subsubsection{SPD Training}

SPD training on the systems focused on working through scripts associated with each use case. In this manner, training was centered on working through the functionality required for each use case with the stakeholders responsible for each role identified in the use cases (see Sections 3.2.1.1, 3.2.2.1, and 3.2.3.1). The training typically took 30 minutes to learn and perform the actions that would be required for SPD use. These actions included: logging on to the system, setting up a WebEx meeting, hosting/joining a WebEx meeting, setting up meeting parameters for collaboration, controlling audio and video parameters, accessing shared files and directories, accessing email, creating whiteboard pages, annotating whiteboards, desktop sharing, annotating shared desktops, and instant messaging. Through this training and dry runs of use cases prior to the Torchlight Parade, the end-users became proficient and all felt well prepared to properly execute their roles. The incident commander was the only exception, he trained quickly on the appropriate use cases the day of Torchlight Parade. Even though his training was limited, the incident commander became proficient in using the system appropriate to his role.

\subsection{Modes of Use}

This section describes three use cases associated with how the LiveWall concept can be incorporated into law enforcement processes as they pertain to a preplanned event. These are presented in chronological order of use:

- Briefing the commander's intent before deploying officers to the event 
- Using the system to increase accuracy and efficiency of the roll call (a notoriously tedious and timeconsuming process). The roll call allows the operations center to confirm that officers scheduled for the event are present and assigned appropriately.

- Using the system to create situation awareness between operations, intelligence, and incident command. This final use case uses any and all of the applications required for each of the respective roles stated above.

\subsubsection{Commander's Intent and Torchlight Briefing}

This section describes how the LiveWall technology can be incorporated into law enforcement processes as they pertain to commander's intent. The commander's intent is a briefing prior to an event that details the desired end-state as it relates to officers, the purpose of the operation, and key tasks to accomplish. The commander's intent effectively informs staff and subordinates of the overall intention of the commander so they may work within those bounds to develop their own plans, orders, and actions. In order to work effectively, it is crucial that intent is properly conveyed to all officers. Prior to the LiveWall effort, the commander's intent would be conveyed to some groups by the commander, and other groups would receive intent later by those who attended the commanders briefing. This was primarily because of logistical constraints of geographic location and available space. The goal of eliminating this gap is twofold: 1) to ensure intent is conveyed uniformly and accurately to all personnel, and 2) to reduce the amount of time required to disseminate the commander's intent. By using the LiveWall concept it is possible for the commander's intent to be observed simultaneously by all personnel even at different geographic locations. This method allowed personnel to observe the commander via audio and video, and observing any content the commander was referencing through the shared desktop on the MondoPad.

\subsubsection{Roles}

The following roles are responsible for acting in the use case for commander's intent and Torchlight Briefing:

Incident Commander - The incident commander is responsible for all aspects of the operation. This includes defining priorities and the overall incident action plan.

SPD Personnel Seattle Justice Center - This refers to LiveWall system and operators located at the Seattle Justice Center, where the system will be used to disseminate commander's intent in real time.

Seattle Police Intelligence Unit - This refers to LiveWall system and operators located at the SPD Intelligence Unit, where the system will be used to disseminate commander's intent in real time.

WebEx Meeting - This refers to the scheduled WebEx session that allows for the collaborative functionality between the actors in this use case.

SPD Personnel at Fire Station \#2 - This refers to LiveWall system and operators located at the Fire Station \#2, where the system will be used to disseminate commander's intent in real time.

SPD Command Van - This refers to the PC tied into the WebEx meeting and operators located in the SPD Command Van. 


\subsubsection{Use Case}

The workflow associated with the commander's intent is detailed in Figure 3.1. As this is the beginning of the Torchlight Parade activities, the system must be set up (steps 1, 2a, 2b, 2c, and 2d in Figure 3.1) and tested (step 3, 3a, 3b, 3c, and 3d in Figure 3.1). After the system is confirmed to be operating properly, the system is ready to execute the actions associated with the focus of the commander's intent use case (steps 4-8 in Figure 3.1). Step 4 in the use case is the primary source for the commander's intent during which the incident commander is using the audio and video of the MondoPad to convey intent and the MondoPad's desktop to work through a Microsoft PowerPoint presentation with information and details associated with the operations for the Torchlight Parade event. All of the other locations can view any manipulation of the desktop and hear and see the incident commander as he discusses operations in real time (steps 5a, 5b, 5c, and 5d). After the commander's presentation, it is possible to open up discussion from remote locations for question and answer session (Q\&A) seen in steps 6-8 in Figure 3.1.

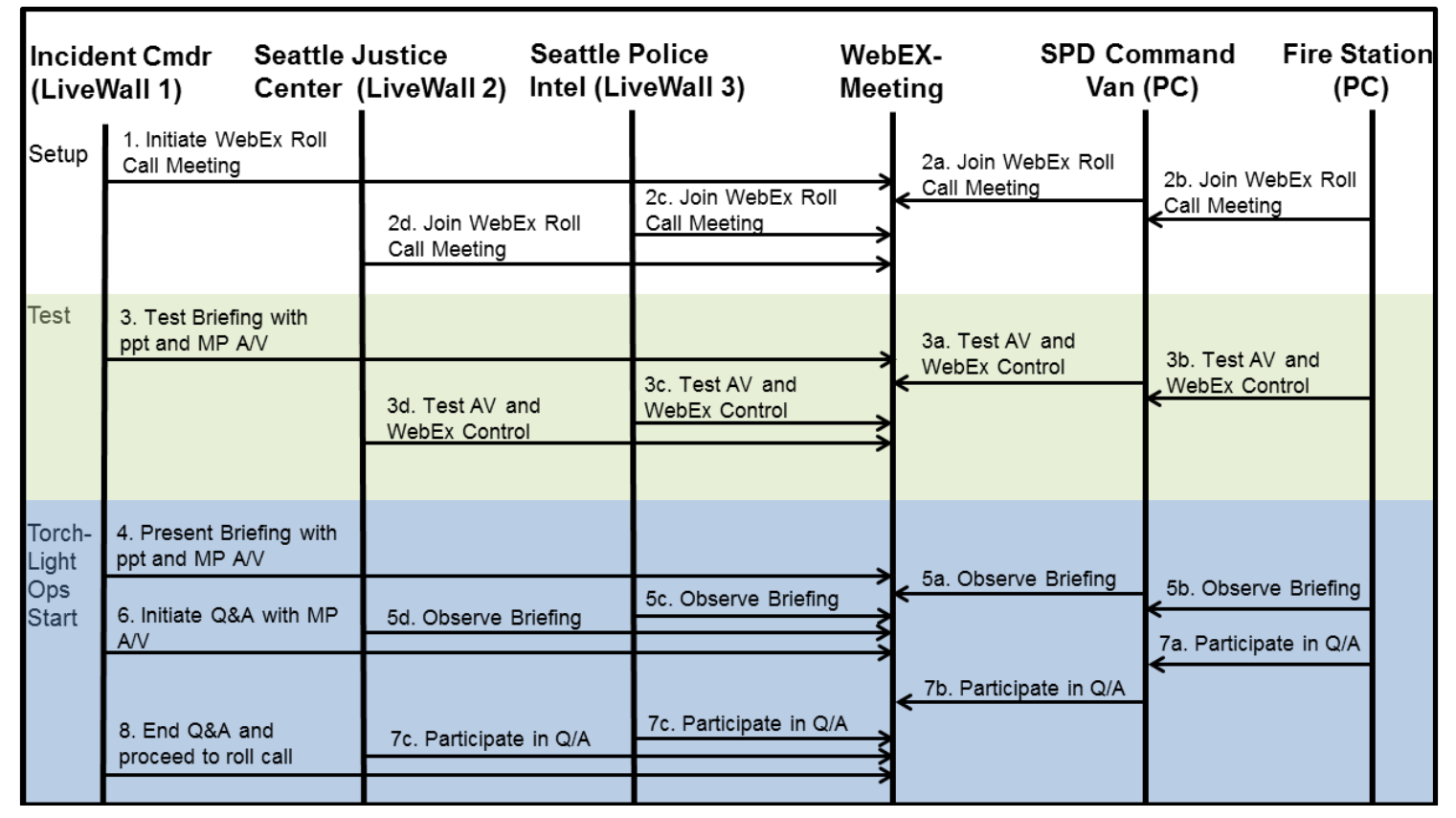

Figure 3.1. LiveWall-based Workflow for Commander's Intent

\subsubsection{Roll Call via LiveWall}

During roll call SPD operations ensures personnel assigned to the event are in attendance and at the proper location. In the case where personnel are absent, operations must work to find coverage for that role. Formal recording of these actions is required for an official document detailing where SPD personnel reported for duty, and for payroll purposes.

\subsubsection{Roles}

West Precinct - This refers to LiveWall system and operators located at the SPD West Precinct, where the system will be used to confirm and record roll call information. 
SPD Personnel Seattle Justice Center - This refers to LiveWall system and operators located at the Seattle Justice Center, where the system will be used to transfer roll call information from Sergeants to the West Precinct.

SPD Share Drive - This refers to a networked share drive to be used exclusively by the LiveWall systems for creating, storing, and organizing files.

WebEx Meeting - This refers to the scheduled WebEx session that allows for the collaborative functionality between the actors in this use case.

SPD Command Van - This refers to the PC tied into the WebEx meeting and operators located in the SPD Command Van.

\subsubsection{Use Case}

Traditionally roll call is accomplished by conducting a paper-based roll call at each location and then having a "runner" bring the roll call sheets to the operations center. At this point the operations center goes over each roll call sheet and transcribes the information to a central document for reporting purposes. With the Roll Call via LiveWall use case the operations center uploads the central document in the form of a spreadsheet file on the share drive and then opens it on the MondoPad with Microsoft Excel. The operations center staff is operating a LiveWall system at the West Precinct and communicates with the Sergeants conducting roll call at the Seattle Justice Center through their LiveWall system and the WebEx meeting which was opened at the beginning of the commander intent use case. The operations center user then verbally read off names and confirms whether they are present or absent with the Sergeants. As the names and status are being read off, the operations user is marking the appropriate category on the spreadsheet which is also viewed by the reporting Sergeants. This process expedites roll call by eliminating the need for runners and the transcription of roll call sheets. It also improves accuracy by removing transcription errors and allowing another user to check the accuracy as the information is being input in front of them via the shared desktop. Figure 3.2 depicts the LiveWall-based work flow for roll call. 


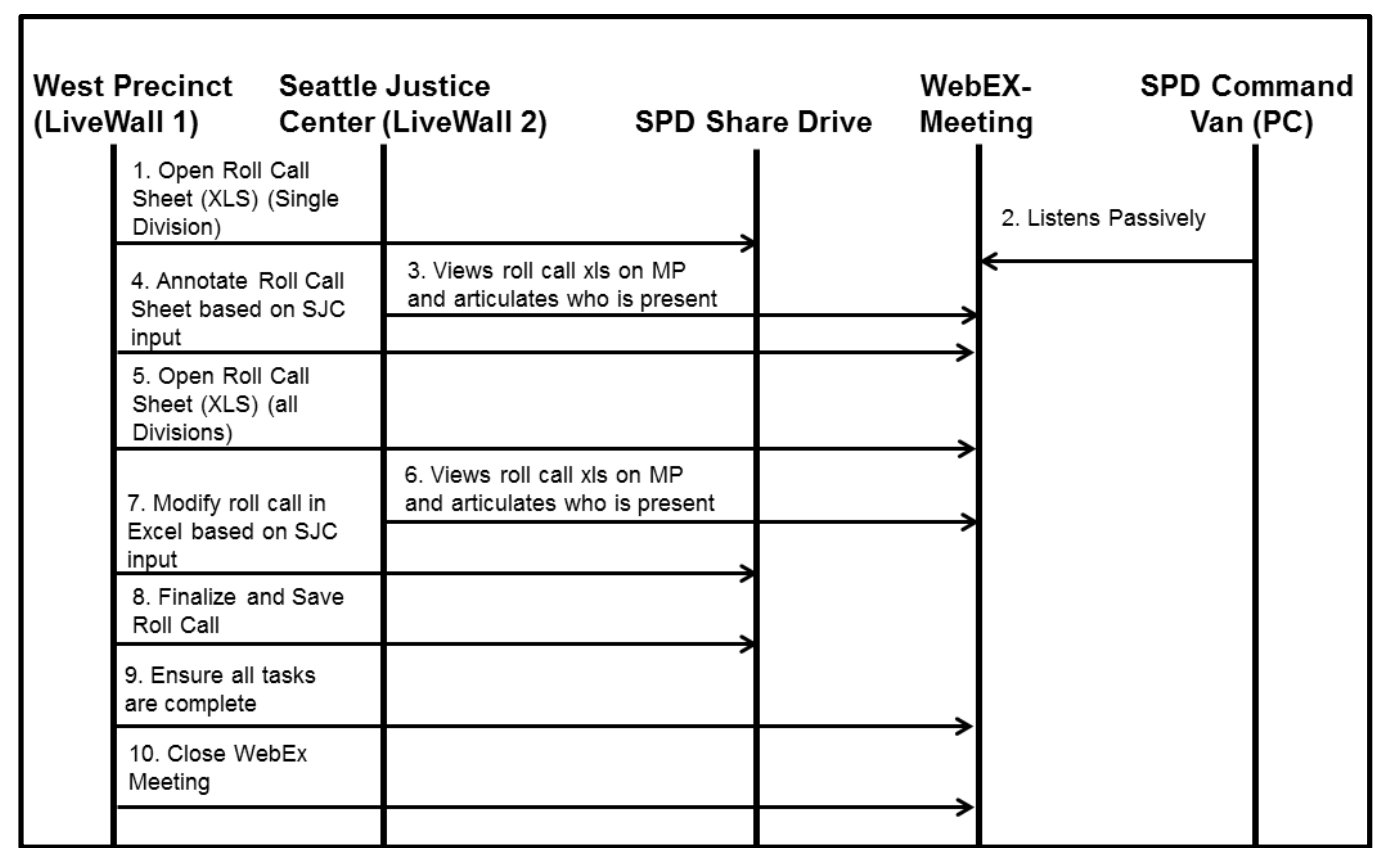

Figure 3.2. LiveWall-based Workflow for Roll Call

\subsubsection{Situational Awareness and Steady State Operations}

Situational awareness is a cognitive state that reflects the current, real-time understanding of an environment and its relation to pertinent goals. A comprehensive understanding of a situation reduces uncertainty during decision making. Common situation awareness underlies effective communication and helps put individual activities in a broader context. Situational awareness has been shown to be significantly related to performance for those who have the technical and operational capabilities. However, it becomes increasingly difficult to maintain good situational awareness as the complexity and dynamics of the environment increase. Technologies assisting with situational awareness need to provide sufficient information to understand both the situation and any change of state without taxing the abilities of humans to act as timely decision makers. Often, an understanding of what is happening outside one group's purview is relevant to a larger understanding of the event and can assist that group in making more informed decisions. An example of the systems being used to share operational information is shown in Figure 3.3.

This use case supports improved situation awareness for operations and response for the primary players associated with SPD's Torchlight Parade operations (incident command, operations, intelligence, and the public information officer). This use case improves situational awareness by:

1. Allowing an always-available audio and video connection between Incident Command, Operations, and Intelligence. This allows for effective briefings as necessary.

2. Allowing each role or user to share the electronic tools and applications they natively use in real time and simultaneously explain the details and significance of what they are displaying to others through the available audio and video link. 
3. Using the desktop sharing, whiteboard, and annotation tools in order to work through a problem, clarify details, and/or brainstorm as a unified team across locations.

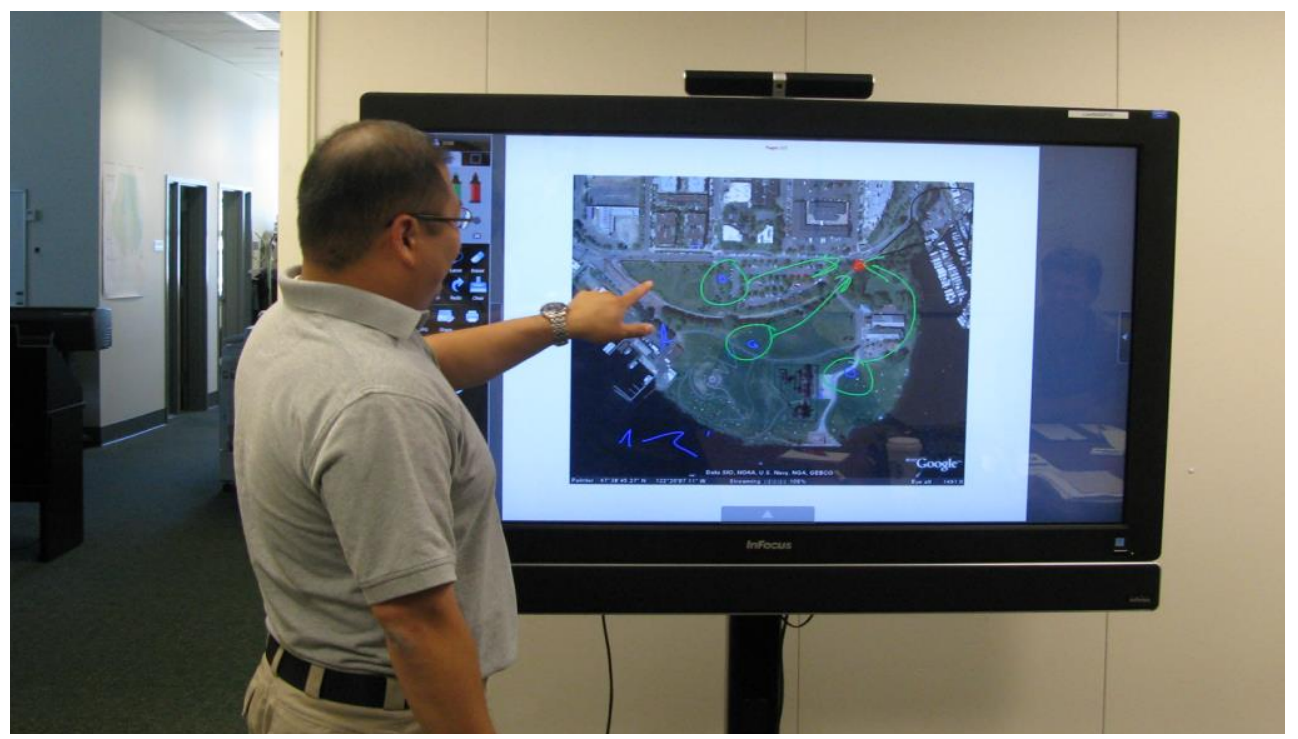

Figure 3.3. Seattle Operations Staff Annotating a Shared Map on a MondoPad

\subsubsection{Roles}

The following roles are responsible for acting in the use case for Situational Awareness and Steady State Use:

Incident Commander - The incident commander is responsible for all aspects of the operation.

Seattle Police Intelligence Unit - This refers to LiveWall system and operators located at the SPD Intelligence Unit, where the system will be used to disseminate intelligence products.

WebEx Meeting - This refers to the scheduled WebEx session that allows for the collaborative functionality between the actors in this use case.

SPD Share Drive - This refers to a networked share drive to be used exclusively by the LiveWall systems for creating, storing, and organizing files.

Seattle Police Operations Center - This refers to LiveWall system and operators located at the SPD Operations Center, where the system will be used to disseminate situation updates, resource-related information, and the demobilization report.

\subsubsection{Use Case}

Traditionally situational awareness would have pertinent details conveyed through radio, phone, and email. Unfortunately, these tools lack the "richness" afforded the simultaneous sharing of audio, video, and desktop. This added richness combined with the available expertise and interaction afforded by the simultaneous audio and video capabilities of the LiveWall system allows for information to be more 
accurately transferred for improved situational awareness and decision support. For the situational awareness use case (Figure 3.4) there were 4 phases of activity:

1. initiate LiveWall operations

2. present, observe, and act on information

3. share and disseminate documents

4. close operations.

Phases 2 and 3 will occur throughout the operations and can occur in any order or even simultaneously.

To begin the workflow for situation awareness, a new WebEx session was required to be initiated by operations and joined by the other attendees. This was because of the sensitivity of the information that can be discussed during the operation. Once all parties had joined the meeting, the bulk of activity would be in Phase 2, Present, observe, and act on information (Figure 3.4). For simplicity this is shown in Figure 3.4 as originating from the Operations Center. However, this action (step 3, Figure 3.5) could originate from any of the meetings' participants, and then the remaining parties would then observe the information, discuss, and act on it (step 4, Figure 3.4). Similarly in Phase 3, Share and disseminate documents, step 5 could originate from other players, and step 6 from the remaining parties. Finally, in Phase 4 the incident commander concludes operations and all parties exit the WebEx meeting.

In Phase 2 the following primary tools were employed for sharing: an Excel document and WebEx whiteboard to detail arrests and arrest status, the operations center's resource map, and screen captures from the intelligence unit. For Phase 3, the primary documents moved through the share drive were annotated maps and camera-based images that were edited and discussed in Phase 2, and then uploaded to the share drive to disseminate to appropriate parties in the field.

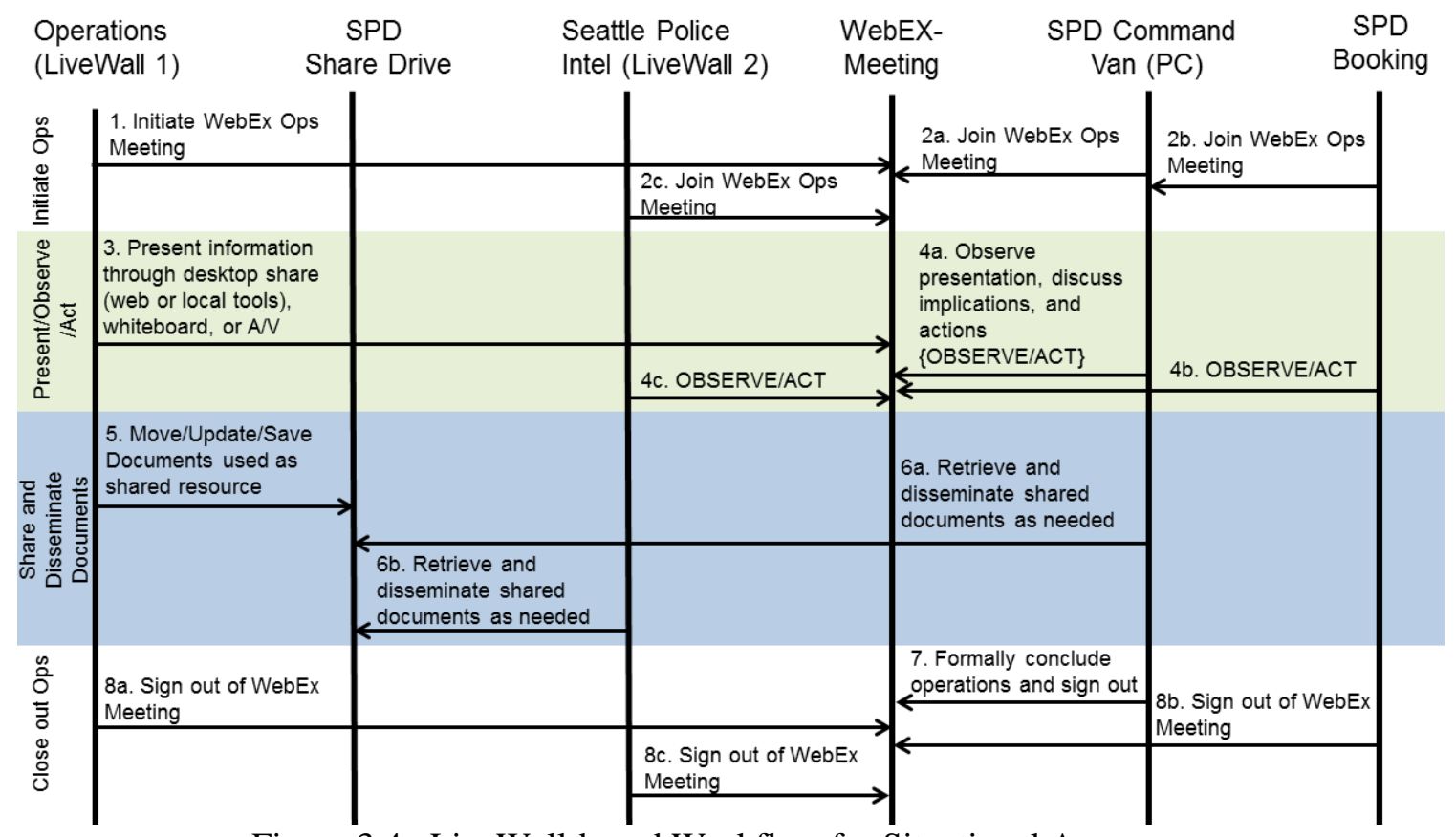

Figure 3.4. LiveWall-based Workflow for Situational Awareness 


\subsection{Operational Evaluation of Law Enforcement Use Cases}

PNNL embedded observers with various user groups to observe and evaluate the LiveWall concept as implemented in the SPD operations for the Torchlight Parade. For the commander's intent use case, two observers were located at the West Precinct with the incident commander, and one observer was located at Seattle Justice Center. For the roll call use case, an observer was located at each of the following locations: West Precinct, Seattle Justice Center, and the Command Van. Finally, during the period where the system was used for situational awareness and steady state use case, there were two observers at SPD's Operations Center and one observer at the Command Van. The evaluations of each portion of the operation are detailed below.

\subsection{Commander's Intent and Torchlight Briefing}

From the vantage point of the West Precinct evaluators the commander's intent use case was executed as anticipated with the video, audio, and presentation all working properly and moving the intent through the WebEx meeting in real time without complications (Figure 4.1). As anticipated, the use of the LiveWall concept ensured intent was conveyed uniformly and accurately to all personnel, and reduced the amount of time required to disseminate the commander's intent, as other supervisors did not have to repeat the message to the officers under their command. From the report from other locations, the intent was conveyed, but with difficulty in portions of the room (e.g., back of the room).

In both the Fire Station and the Seattle Justice Center, the sound level was not adequate to have the content easily heard. Although both of these locations were tested with the MondoPad system at the Seattle Justice Center and the PC at the Fire Station, neither test was conducted with a full assembly in attendance. Both the increased level of background noise and the sound attenuation associated with the number of bodies in both locations are the primary differences between the system in testing and the system in operation. It was concluded that the audio setup of the systems were not adequate to project the commander's intent at the levels desired by the attendees. This can be resolved by determining how to better integrate with the audio/visual (AV) systems in the room, supplying appropriately powered audio equipment in locations without dedicated in-room AV systems, and document and train users to set the systems up according best practices gleaned from testing.

The evaluators, with concurrence from SPD, concluded that this use case was a success and could increase law enforcements effectiveness and save considerable time during pre-planned event operations. It is anticipated that this use case would be very effective in disseminating commander's intent during unplanned and unfolding situations such as an earthquake, unanticipated large-scale protests, or terrorist attack. In these situations, it may be argued, that time savings are even more critical and will allow law enforcement to respond in a shorter amount of time and with more clarity of purpose. 


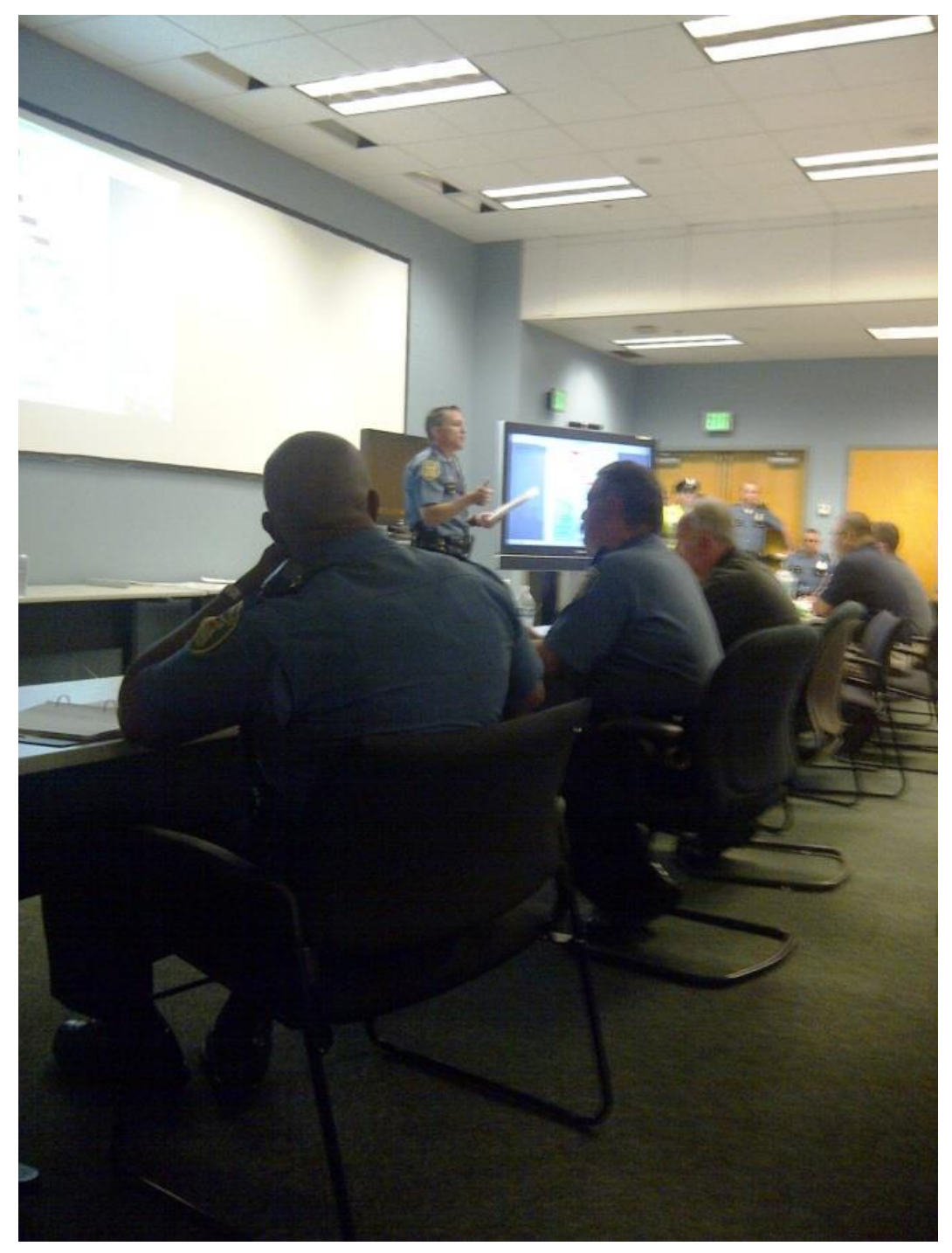

Figure 4.1. Incident Commander Shown Simultaneously Briefing Commander's Intent with LiveWall System to Officers at Local and Remote Locations

\subsection{Roll Call via LiveWall}

The users at operations and the precincts supporting the Torchlight Parade expedited roll call through the use of the LiveWall system. Again, the use of the LiveWall concept ensured information was communicated and recorded accurately and reduced the amount of time required to conduct roll call. The estimate of time savings from operations was roughly 2 hours saved for a full roll call by using the system. The use of the system for roll call was not without shortcomings. The preferred method of recording information was through shared annotation on top of an excel spreadsheet. However the permissions associated with annotation took some time to grant as they were not set up prior to the start of roll call. It was also learned that the accidental selection of "stop annotation" cleared all annotations on the spreadsheet, which required that the information be reentered. These concerns could easily be addressed by allowing users to directly access the desktop, and therefore the roll call spreadsheet, on the operations MondoPad. It was suggested that an ideal solution would be to create an Excel sheet with 
macros that would allow for touch-based input for present or absent. PNNL feels that for many interactive uses such as roll call, touch-screen-adapted applications and documents would be well worth the effort to convert in order to further expedite processes.

The evaluators, with concurrence from SPD, declared this use case as a success. Even with the problems experienced by users, the time savings was still significant and the accuracy of the roll call was guaranteed. The concerns of annotation on a detailed document could be mitigated through the direct interaction with the document in the future.

\subsection{Situation Awareness and Steady State Operations}

A variety of information was shared during this phase of the operation. A test scenario was run during Torchlight Parade whereby the LiveWall locations were able to share and collaborate on fictitious suspect information simultaneously. For this effort an image was uploaded to LiveWall, where LiveWall users collaborated on identifying the location of suspect and potential threats (e.g., weapon in pocket). The completed annotated picture was pushed to mobile devices in the field through LiveWall. This process was fairly intuitive and seamless to the both the intelligence and operations users. It is anticipated that if a similar threat were encountered during operations it could have been handled in a similar manner. Additionally, for the first time SPD was able to share arrest report data in near-real time with Incident Command and the Public Information Officer without using runners to update and share the information.

The Situational Awareness and Steady State Operations use case was evaluated as a qualified success. Important operational information was moved through the LiveWall systems and was able to be both acted upon and disseminated but the amount of use and information density was sparse resulting in reduced use from what was anticipated for this phase. There are a number of potential reasons for this. The first being that the MondoPad's were placed in locations where there was available space and adequate separation from general SPD personnel in order to share sensitive intelligence information. This separation also made using the LiveWall systems more of an effort as they were located in spaces outside of other workstations (both for intelligence and operations) and would require users to leave their workstation to go "check" the system.

The second reason relates to information density available through the LiveWall concept. As this was the first pilot for the technology, and it was only available to SPD, there was not as much information available through the system as would be desired. Desired information that was not available falls into three categories:

- additional SPD data and applications (e.g., geo-spatial situational awareness application being tested by SPD, Blue Force, or police officer, tracking),

- Shared data (e.g., camera feeds)

- Communication and information from other agencies pertinent to SPD and vice versa.

An example to illustrate this last point, overall command was with SPD so when issues arose, the leads for the various agencies would go to the Command Van and report the issue and actions taken. There were dedicated SPD personnel in the Command Van who documented on paper the time and description of each issue and the actions taken. Much of this interaction could have occurred with those agencies commanders communicating and documenting information through a LiveWall type system. 


\subsection{Summary and Recommendations}

The Seattle Law Enforcement Pilot for the LiveWall concept was deemed a success from both the PNNL evaluators and the stakeholders from SPD (operations, intelligence, and information technology). The system demonstrated the ability to

- substantially reduce amount of time for various tasks as seen when disseminating commander's intent, performing roll call, disseminating arrest information to operations and public information officer

- improve accuracy with which information is transferred as seen in disseminating the commander's intent and jointly working through roll call

- support improved situation awareness as seen when collaborating on potential threats.

SPD is planning on keeping the LiveWall systems installed in operations and is working to use the systems in more day-to-day activities such as briefings and other meetings. SPD staff felt that the more familiarity that users had with the system, the more they would be comfortable when using it and understanding the strengths it affords.

The LiveWall system as deployed for Torchlight Parade could have been improved in a number of ways. The first being a more thorough testing of systems to ensure that both audio and video is environmentally appropriate when presentations are made to moderate to large audiences during operations. The second would be to co-locate the LiveWall Systems with SPD users performing their normal functions rather than sequestering them in rooms with little traffic or personnel. The concern for how to limit the remote audience for sensitive information will either need to be investigated and addressed with a technology and/or policy-based solution. SPD also desired some additional features associated with the LiveWall. These features included an always available meeting room(s) and the ability to overlay semi-transparent full screen video with the shared desktop. These desired features were presented to a number of vendors and developers, including a team from InFocus, the maker of the MondoPad.

PNNL has concluded that for future deployments of LiveWall, the participants should take into account the following:

- Technology-averse users can be brought into the fold by expanding the concept of operations to include tasks associated with daily operations.

- LiveWall concept should be formally extended to patrol via handheld devices in order to improve situation awareness for all parties.

- Modifying documents to take advantage of LiveWall's touch screen modality would yield a significant time saving and increase adoption.

- There is a need to develop formalized requirements for LiveWalls used specifically in large briefing situations. The requirements collected will be focused on closing the gap between a live meeting and a LiveWall meeting. 



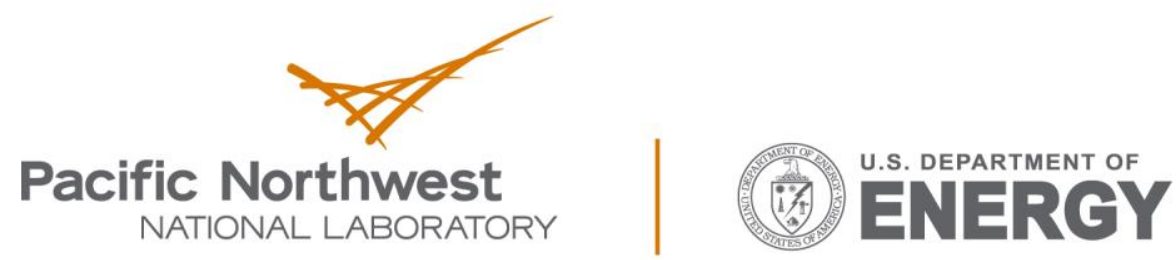

Proudly Operated by Battelle Since 1965

902 Battelle Boulevard

P.O. Box 999

Richland, WA 99352

1-888-375-PNNL (7665)

www.pnnl.gov 